Corrigendum

\title{
Corrigendum to "Intelligent Techniques Using Molecular Data Analysis in Leukaemia: An Opportunity for Personalized Medicine Support System"
}

\author{
Haneen Banjar $\mathbb{D}^{1,2}$ David Adelson $\mathbb{D}^{1},{ }^{3}$ Fred Brown, ${ }^{2}$ and Naeem Chaudhri ${ }^{4}$ \\ ${ }^{1}$ Department of Computer Science, King Abdulaziz University, Jeddah, Saudi Arabia \\ ${ }^{2}$ School of Computer Science, University of Adelaide, Adelaide, SA, Australia \\ ${ }^{3}$ School of Molecular and Biomedical Science, University of Adelaide, Adelaide, SA, Australia \\ ${ }^{4}$ Oncology Centre, Section of Hematology, HSCT, King Faisal Specialist Hospital and Research Centre, Riyadh, Saudi Arabia \\ Correspondence should be addressed to Haneen Banjar; hrbanjar@kau.edu.sa
}

Received 2 May 2018; Accepted 9 May 2018; Published 21 June 2018

Copyright (C) 2018 Haneen Banjar et al. This is an open access article distributed under the Creative Commons Attribution License, which permits unrestricted use, distribution, and reproduction in any medium, provided the original work is properly cited.

In the article titled "Intelligent Techniques Using Molecular Data Analysis in Leukaemia: An Opportunity for Personalized Medicine Support System" [1], there was an error in the order of the first and second affiliations. The corrected affiliations' list is shown above.

\section{References}

[1] H. Banjar, D. Adelson, F. Brown, and N. Chaudhri, "Intelligent techniques using molecular data analysis in leukaemia: An opportunity for personalized medicine support system," BioMed Research International, vol. 2017, Article ID 3587309, 12 pages, 2017. 


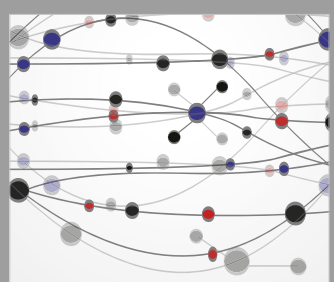

The Scientific World Journal
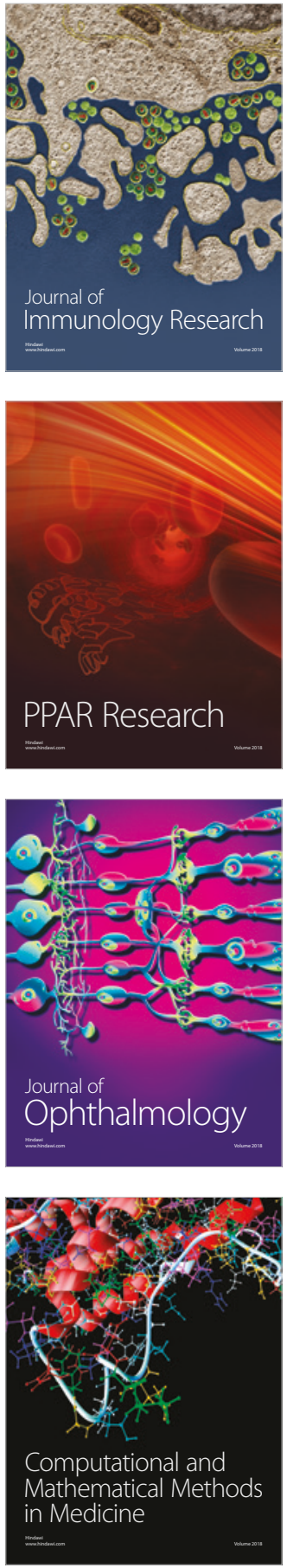

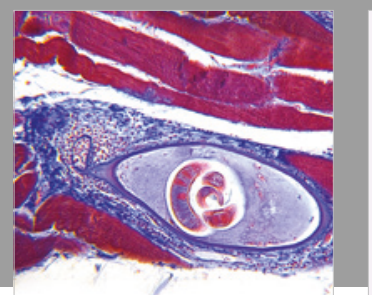

Gastroenterology Research and Practice

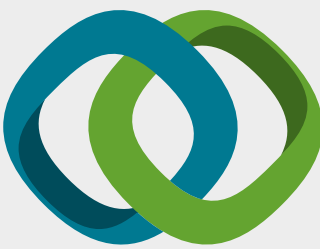

\section{Hindawi}

Submit your manuscripts at

www.hindawi.com
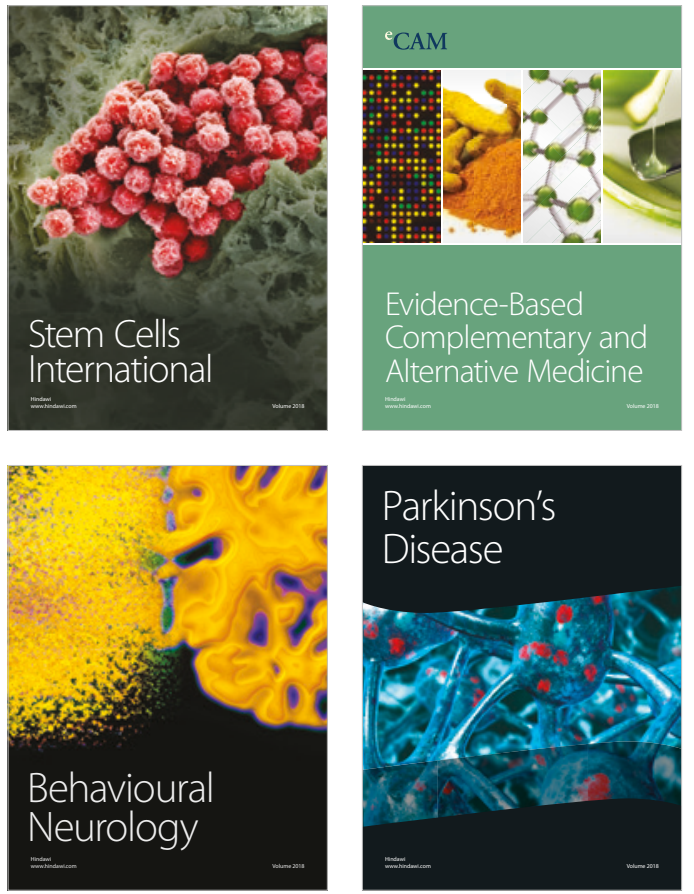

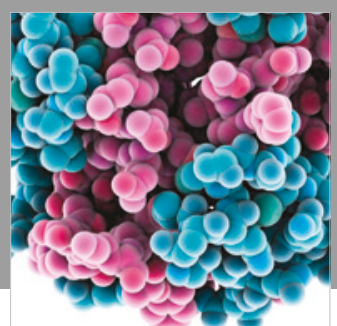

ournal of

Diabetes Research

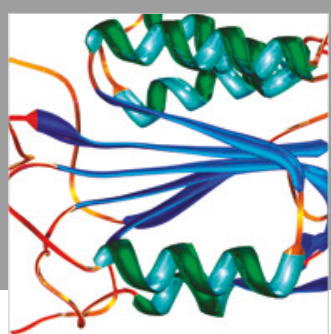

Disease Markers
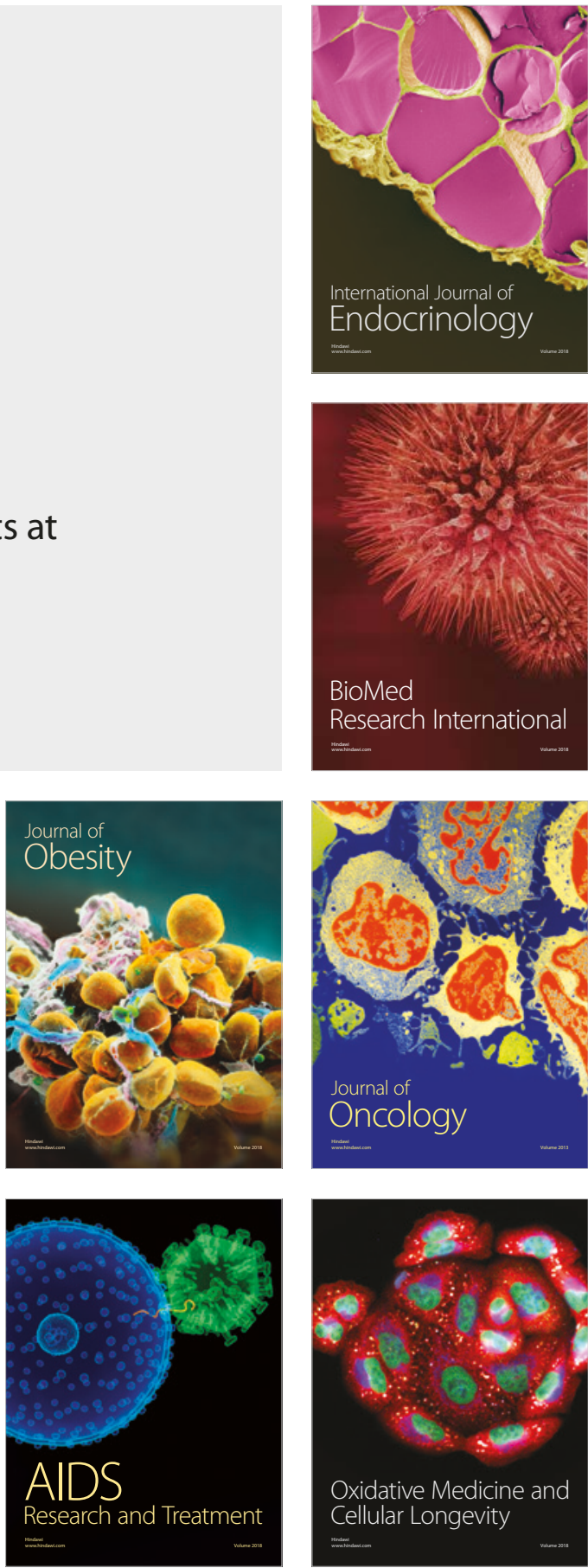